\title{
HRTEM Determination of Cubic GaN Formation in MBE Grown Mn/GaN Films
}

\author{
V. K. Lazarov, M. M. Goetz, D. P. Robertson, Y. Cui, H. Liu, L. Li and M. Gajdardziska-Josifovska
}

Department of Physics and Laboratory for Surface Studies, University of Wisconsin-Milwaukee, P.O. Box 413, Milwaukee, Wisconsin 53201

Group-III nitride semiconductors have attracted ample attention due to their applications in blue lasers, light emitting diodes, high temperature electronics and spintronics. The energy gap of $2 \mathrm{H}$ wurtzite nitrides covers the entire visible spectrum, motivating work on bandgap engineering via controlled growth of AlGaInN alloys. Group-III nitrides also crystallize in $3 C$ zinc blende structure with very different physical and electronic properties. Hence an alternative route to bandgap engineering may be through synthesizing superlattices of hexagonal $(h)$ and cubic $(c)$ polytypes with controlled modulation of the superlattice periodicity. If such superlattice structures can be materialized, a new class of materials can be created with controllable bandgap, while evading the phase separation problem that limits bandgap engineering through composition modulation. We have recently demonstrated the first synthesis of an $h-G a N / c-G a N / h-G a N$ heterostructure, inducing the growth of the cubic phase by Mn multilayer delta doping [1]. Here we report the microscopy study that enabled the determination of this structure.

$\mathrm{GaN}$ heterostructures were grown on $6 \mathrm{H}-\mathrm{SiC}(0001)$ by electron-cyclotron resonance plasma-assisted molecular beam epitaxy (MBE). The growth process consisted of first depositing $20 \mathrm{~nm} \mathrm{GaN} \mathrm{buffer,}$ followed by ten periods of alternating layers of 1 monolayer (ML) Mn and $10 \mathrm{ML}$ GaN, and finally capped with $30 \mathrm{~nm}$ GaN. Cross sectional TEM samples were prepared by conventional cutting, mechanical polishing, dimpling and Ar ion milling methods. All TEM images were obtained using a $300 \mathrm{KV}$ Hitachi H9000-NAR microscope with a point resolution of $0.18 \mathrm{~nm}$ and lattice resolution of $0.11 \mathrm{~nm}$ in the phase contrast high-resolution (HRTEM) imaging mode. Digitally recorded HRTEM images were analyzed by numerical diffractograms to quantitatively determine the lattice structures of the different film layers, as illustrated in Fig. 1. It was found that the crystal structure in the GaN buffer and cap layers match the $2 \mathrm{H}$-wurtzite GaN. However, uniform cubic zinc blende GaN phase was observed in place of the nominal multilayer $\mathrm{Mn} / \mathrm{GaN}$ region. The density of defects typically observed in GaN films was drastically reduced within the cubic and cap layer, indicating improved film quality possibly due to the surfactant effect of Mn.

A critical question is if $\mathrm{Mn}$ is indeed responsible for the cubic GaN phase observed in the $\mathrm{Mn} / \mathrm{GaN}$ region, or if this phase is akin to the cubic inclusions that are occasionally found in hexagonal GaN films. To address this concern, we grew a structure that consists of a single Mn layer sandwiched between two GaN layers $40 \mathrm{~nm}$ and $100 \mathrm{~nm}$ thick using the same growth conditions. Detailed diffractogram analysis of the HRTEM images (not shown here) revealed only $2 \mathrm{H}$-wurtzite GaN, and no cubic phase was detected. Again, there was no visible Mn monolayer and the density of defects was clearly reduced beyond the nominal position of the Mn layer deposition, confirming the surfactant effect of Mn. This experiment strongly suggests that the cubic GaN observed in the multilayer samples is unlike the small inclusion domains seen in previous studies. It also strongly indicates that the periodic insertion of Mn layer during MBE growth is necessary to promote the formation of cubic $\mathrm{GaN}$. 
[1] Y. Cui et al., Appl. Phys. Lett. (submitted).

[2] Research supported by NSF/DMS grants 9553148 and 0094105.
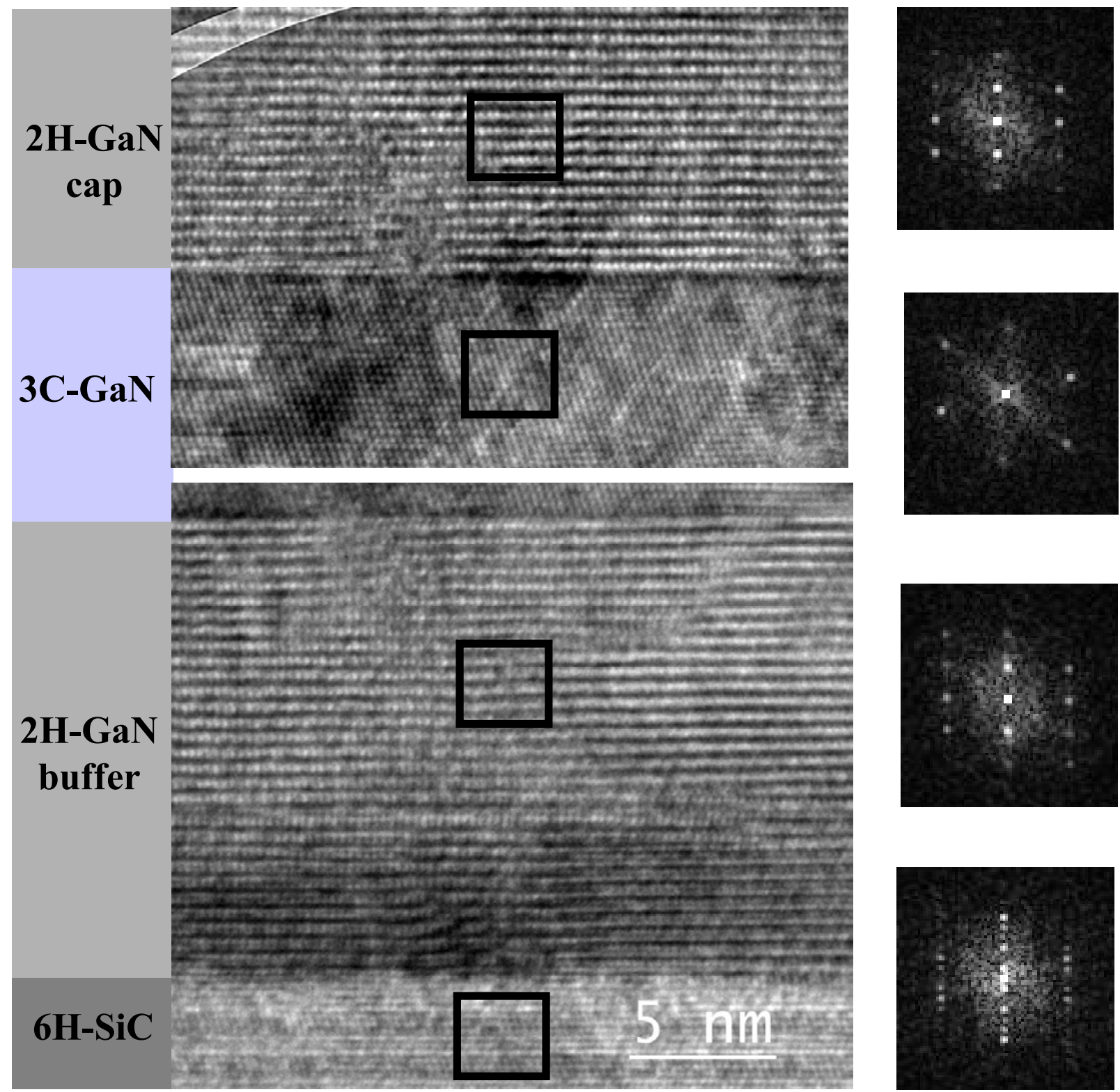

FIG. 1. HRTEM micrographs recorded with electron beam parallel to [11 $\overline{2} 0]$ zone of $6 \mathrm{H}-$ $\mathrm{SiC}(0001)$ substrate. Numerical diffractograms, shown on right, were calculated by fast Fourier transforms of areas marked by box in each layer. Lattice spacing and inter-planar angle measurements allowed phase determination of GaN heterostructure as follows: hexagonal wurtzite $2 \mathrm{H}-\mathrm{GaN}$ buffer, cubic zinc blende $3 \mathrm{C}-\mathrm{GaN}$ in $\mathrm{Mn}$ multilayer doped region, and hexagonal wurtzite $2 \mathrm{H}-\mathrm{GaN}$ capping layer. 